\title{
Flutter of Winged Reentry Space Vehicles Affected by an Elastic Attachment in Launching Configuration
}

\author{
By Atsushi KANDA and Tetsuhiko UEDA \\ National Aerospace Laboratory of Japan, Tokyo, Japan
}

(Received July 23rd, 2003)

\begin{abstract}
This paper reports the flutter investigation of a winged reentry space vehicle having rotational modes in dynamic deflection due to an elastic attachment between a vehicle and a booster rocket. The elastic rotational mode is taken into consideration as an elastic rolling mode or an elastic yawing mode. Flutter experiments have been conducted in a transonic wind tunnel. The doublet-point method (DPM) is used to calculate flutter boundaries for this model. It is shown that an elastic rolling mode may lower the critical speed of anti-symmetric mode flutter because its existence alters the natural vibration mode of anti-symmetric bending which causes flutter. On the other hand, a coupling between an elastic yawing mode and an anti-symmetric bending one becomes critical in the different model.
\end{abstract}

Key Words: Flutter, Yaw, Roll, Wind Tunnel Test, Doublet-Point Method, Reentry Vehicle

\section{Introduction}

The winged reentry space vehicle project, H-II Orbiting Plane Experimental (HOPE-X), is under consideration in Japan. The HOPE-X vehicle will be attached atop of a launching rocket. In this case, the vehicle has natural vibration modes due to an attachment between the rocket and the vehicle (modes due to a launching rocket itself may be included). The object of this research is to investigate flutter characteristics of the vehicle affected by elastic modes due to an attachment.

Such elastic modes are rotational as a rolling, pitching and yawing mode from a vehicle standpoint (Fig. 1). A significant amount of research has been conducted on flutter characteristics affected by rotational modes of a vehicle. In the Active Flexible Wing (AFW) project, ${ }^{1)}$ cooperated between Rockwell International and NASA Langley Research Center, an anti-symmetric mode flutter of the flexible wing with a roll free motion was investigated. Soistmann and Spain $^{2)}$ researched the flutter of a lifting body with a pitching degree of freedom. Chen and Dugundji ${ }^{3)}$ analyzed the flutter of a forward swept wing made of composite materials with a pitching degree of freedom. Niblett ${ }^{4)}$ analytically researched a role of a pitching degree of freedom in flutter. These investigations assumed that a vehicle had rotational degrees of freedom in a free flight condition. There has been no investigation on a flutter coupling with rotationally elastic modes except for the investigation of elastic pitching mode effects on flutter by Kanda and Ueda.,6)

We will focus our attention on a rolling mode and a yawing one due to an elastic attachment. Two different supporting systems for wind tunnel tests have been developed. Each system allows an elastic rolling or an elastic yawing mode to a wind tunnel model, which is necessary to conduct wind tunnel tests for the flutter. Flutter experiments are conducted

(C) 2004 The Japan Society for Aeronautical and Space Sciences

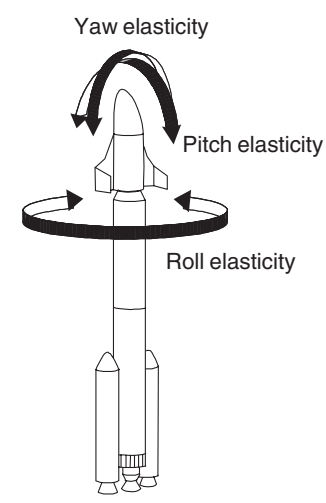

Fig. 1. Launching configuration of re-entry space vehicle.

in the transonic wind tunnel at the National Aerospace Laboratory of Japan (NAL) using these supporting systems. In addition, flutter characteristics are numerically computed by the doublet-point method (DPM). ${ }^{7)}$

\section{Effects of Elastic Rolling Mode}

In this section, flutter characteristics affected by an elastic rolling mode will be presented. Flutter characteristics will be obtained by both wind tunnel tests and analysis. The test is conducted with a full-span model attached to the newly made supporting system which provides an elastic rolling mode to the model simulating the launching configuration. The DPM is employed to calculate unsteady aerodynamics, where the mode shapes for the calculation were tuned with the results of modal tests.

\subsection{Supporting system and wind tunnel model}

\subsubsection{Supporting system}

The original supporting system allowing roll motion ${ }^{8)}$ has been improved to include roll springs for this study. This configuration is shown in Fig. 2. As is clear from Fig. 2, 


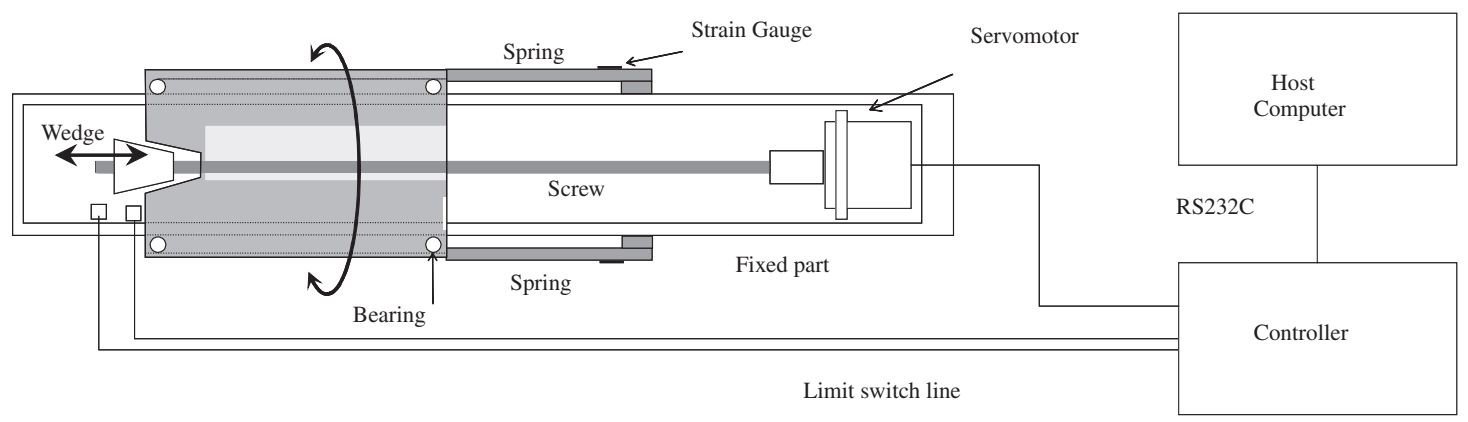

Fig. 2. Schematic of supporting system with elastic rolling mode.

the rotating part to which the model is fitted is able to rotate with ball bearings around the support. The roll springs provide restoring forces to the model in roll. Five springs (A-E) with different stiffness are provided and they can easily be changed. Strain gauges are attached on the springs. The model without springs has a rolling degree of freedom in place of an elastic rolling mode. This supporting system can control the constraint in a rolling degree of freedom. If the wedge illustrated in the figure moves along the axis limiting the rotation, the model also becomes fixed about the axis. The function works as a flutter suppressor because it raises the flutter speed by decoupling the flutter mode.

\subsubsection{Wind tunnel model}

The wind tunnel model (elastic rolling model) is shown in Fig. 3. The wing has a tip-fin configuration. The HOPE-X project had planned this wing configuration in the initial stage. It consists of a main wing and tip-fins. The frame of the model wing is made of the aluminum alloy 7075-T651 and covered by foaming to have the NACA0010 airfoil. Strain gauges are installed on the root of the wing to measure the bending and torsional strain during tunnel tests.

The fuselage has a length of $677 \mathrm{~mm}$ and both a height and width of $160 \mathrm{~mm}$. The main wing has a $200 \mathrm{~mm}$ span, a $400 \mathrm{~mm}$ chord length at the root and $120 \mathrm{~mm}$ at the tip. There are kinks at the leading and trailing edges at $80 \mathrm{~mm}$

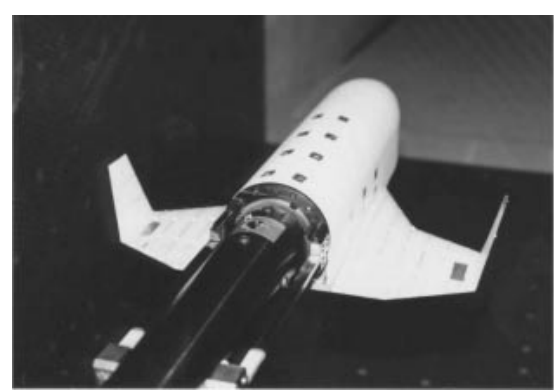

Fig. 3. Elastic rolling model.

in span. The tip-fins have cant angles of $14^{\circ}$, a span length of $120 \mathrm{~mm}$, and a chord length of $60 \mathrm{~mm}$ at the tip. The half wing weighs $1.3 \mathrm{~kg}$.

\subsection{Modal characteristics}

\subsubsection{Modal tests}

The Dynamic Displacement Measurement System ${ }^{9,10)}$ has been used at the modal tests. The system can automatically measure the dynamic displacement (Fig. 4). Natural vibration modes have been calculated from the measured data of 82 points ( 25 on the half main wing and 16 on each tip-fin). The model was excited by an electromagnetic shaker with white noise. The result of the experiment is shown in Table 1 in the case of no spring, spring A and spring D for

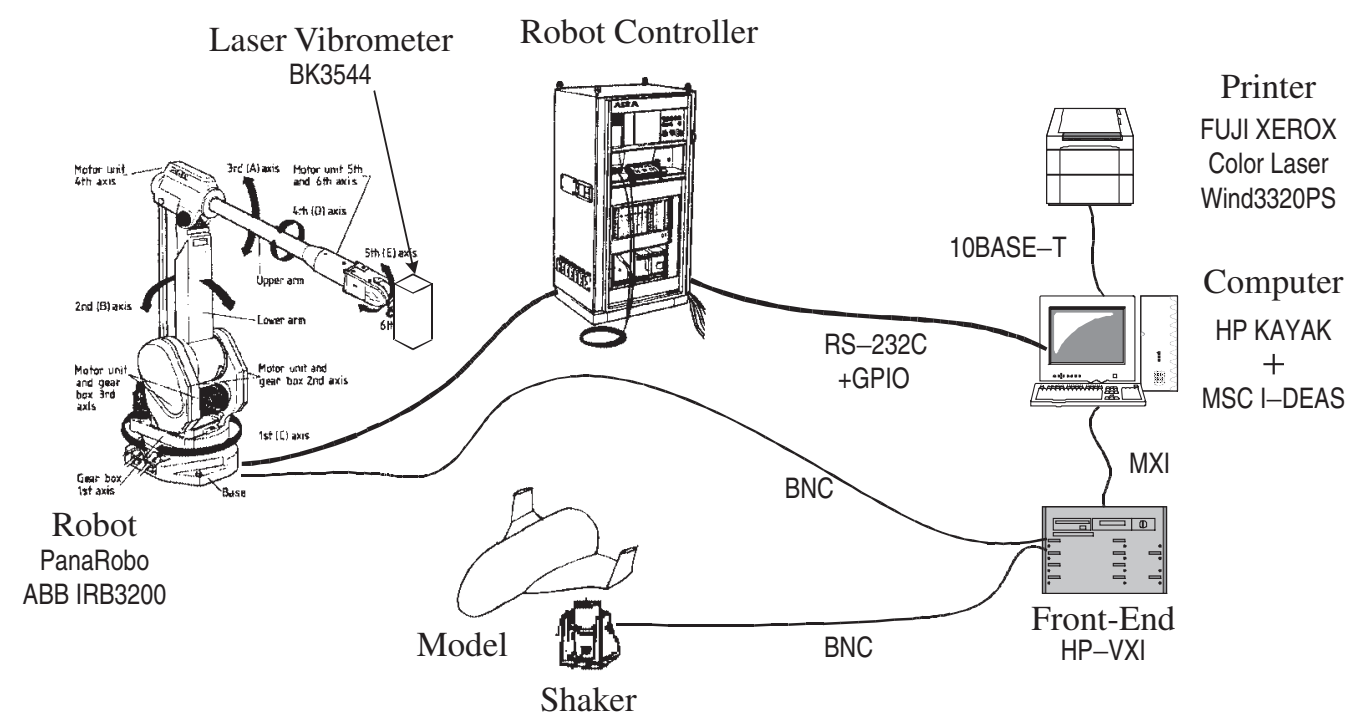

Fig. 4. Dynamic Displacement Measurement System. 
Table 1. Modal characteristics of elastic rolling model (experiment).

\begin{tabular}{|c|c|c|c|c|}
\hline \multirow{2}{*}{ Number } & \multicolumn{3}{|c|}{ Frequency [Hz] } & \multirow{2}{*}{ Mode } \\
\hline & Spring A & Spring D & $\mathrm{W} / \mathrm{O}$ spring & \\
\hline 1 & 21 & 40 & - & Elastic rolling \\
\hline 2 & 67 & 67 & 67 & 1 st symmetric bending \\
\hline 3 & 81 & 86 & 80 & 1st anti-symmetric bending \\
\hline 4 & 97 & 97 & 97 & 1st symmetric torsion \\
\hline 5 & 98 & 98 & 98 & 1st anti-symmetric torsion \\
\hline 6 & 205 & 205 & 206 & 2nd symmetric torsion \\
\hline 7 & 208 & 208 & 208 & 2nd anti-symmetric torsion \\
\hline 8 & 254 & 254 & 254 & 2nd symmetric bending \\
\hline
\end{tabular}

Table 2. Modal characteristics of elastic rolling model (analysis).

\begin{tabular}{|c|c|c|c|c|}
\hline \multirow{2}{*}{ Number } & \multicolumn{3}{|c|}{ Frequency $[\mathrm{Hz}]$} & \multirow{2}{*}{ Mode } \\
\hline & Spring A & Spring D & W/O spring & \\
\hline 1 & 21 & 40 & 0.5 & Elastic rolling \\
\hline 2 & 69 & 69 & 69 & 1st symmetric bending \\
\hline 3 & 82 & 87 & 81 & 1st anti-symmetric bending \\
\hline 4 & 107 & 107 & 107 & 1st symmetric torsion \\
\hline 5 & 108 & 108 & 108 & 1st anti-symmetric torsion \\
\hline 6 & 246 & 246 & 246 & 2nd symmetric torsion \\
\hline 7 & 247 & 247 & 247 & 2nd anti-symmetric torsion \\
\hline 8 & 286 & 286 & 286 & 2nd symmetric bending \\
\hline
\end{tabular}

the supporting system. It should be noted that spring D is stiffer than A.

\subsubsection{Modal analysis}

The result of the FEM analysis is shown in Table 2. The FEM model comprises beam elements and has been tuned with the results of the modal tests.

\subsubsection{Discussion}

If we compare the results of the analysis with those of the tests, they agree well to each other with the natural frequencies of the 1st, 2nd and 3rd mode. On the other hand, the analysis becomes 10-20\% higher than the tests above the 4th mode. The simplification in the FEM model is thought to cause the difference. However, natural vibration modes of the analysis reasonably agree with those of the tests over all modes. Note that the difference in rolling stiffness can be attributed to the natural frequencies of the 1 st and 3rd mode. The natural frequencies of the 1 st and $3 \mathrm{rd}$ mode increase as the stiffness is increased.

Flutter analysis requires the natural vibration modes and natural frequencies. They can be obtained from the modal tests. Natural vibration modes calculated by the FEM are used in flutter calculations because it is difficult to measure the fine modes in the tests. The correlation between the analysis and the test result is fairly good as shown above, whereas the natural frequencies are slightly different in higher order modes. The difference is permissible since the flutter occurs generally by coupling in lower modes. ${ }^{11)}$ It is preferable, however, to use the natural frequencies of the test results instead of the analytical frequencies.

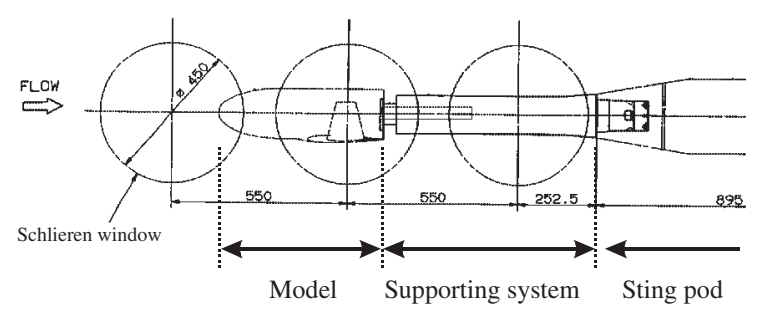

Fig. 5. Wind tunnel test.

\subsection{Flutter characteristics}

\subsubsection{Wind tunnel experiments}

The flutter experiment was conducted in the transonic wind tunnel (the measurement section is $2 \mathrm{~m} \times 2 \mathrm{~m}$ ). The model installed in the tunnel is illustrated in Fig. 5. Flutter search for the boundary is made while the total pressure $P_{0}$ was increased step by step at each Mach number 0.6, 0.7, 0.8 and 1.2. The springs A and D were used. As a result, the flutter speeds are obtained at each Mach number and $M=0.92$ and 0.96 as well. Figure 6 shows the time histories of the strain at $M=0.6$ on the spring A. The left figures show the histories from the onset of flutter to its suppression. The right part shows the time histories of flutter. We can recognize from these histories that the flutter has an anti-symmetric mode with respect to the plane of symmetry and it could be suppressed by utilizing the function of the supporting system.

\subsubsection{Flutter analysis}

Flutter characteristics are calculated ${ }^{6)}$ using the DPM and the $\mathrm{p}-\mathrm{k}$ method. The anti-symmetric deformation and the 


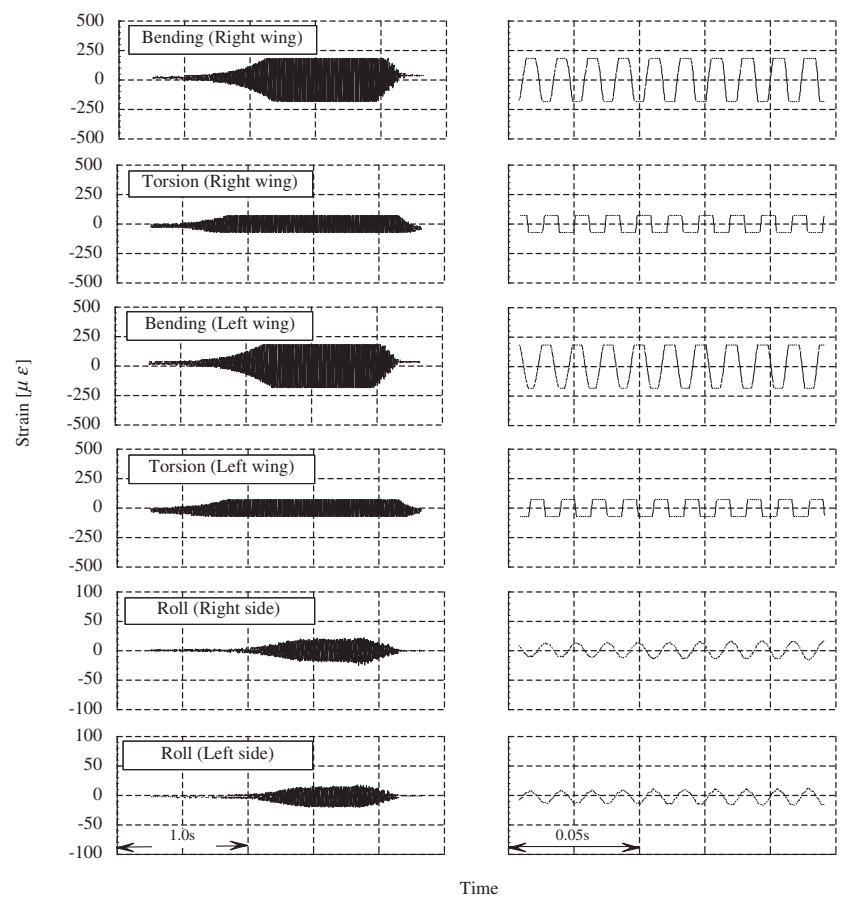

Fig. 6. Time history of anti-symmetric mode flutter.

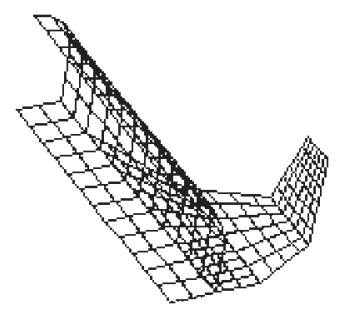

Fig. 7. Aerodynamic model.

symmetric one can be separated in the linear theory, saving computation time. The 1st, 3rd, 5th and 7th modes are used to compute for anti-symmetric flutter and the 2nd, 4th, 6th and 8th modes for symmetric flutter. Mach numbers are selected at $M=0.6,0.7,0.8,1.2$ and 1.5 . The aerodynamic panels of the right half of the wing consist of 196 elements ( 60 for the main wing, 46 for the tip-fin and 100 for the fuselage), as shown in Fig. 7.

Flutter boundaries are calculated by the $\mathrm{p}-\mathrm{k}$ method with a structural damping of 0.03 . If the flow is assumed to be inviscid, irrotational and isentropic, the non-dimensional flutter speed is defined by Eq. (1),

$$
\overline{U_{F}}=\frac{1}{2 \pi f_{\alpha} b} \sqrt{\frac{\pi b S_{\mathrm{w}}}{2 m}} \sqrt{\frac{\gamma P_{0} M^{2}}{1+(\gamma-1) M^{2} / 2}}
$$

where $m$ is the wing mass, $b$ is a half chord of the main wing at the root, $S_{\mathrm{w}}$ is the wing area, $f_{\alpha}$ is the natural frequency of the torsional mode and $\gamma$ is the specific heat ratio.

As a result, the anti-symmetric mode flutter that couples with the 3 rd and 5 th modes becomes critical. The symmetric flutter coupling with the 2nd and 4th modes is also obtained. As an example, the analytical result of the anti-symmetric flutter at $M=0.6$ with the spring A is shown in Fig. 8 .
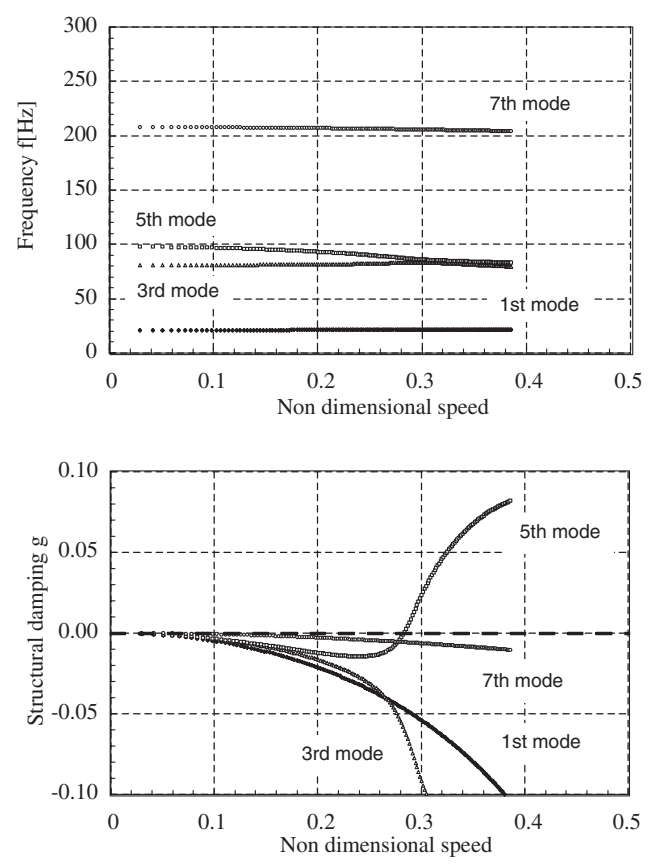

Fig. 8. Anti-symmetric mode flutter analysis.

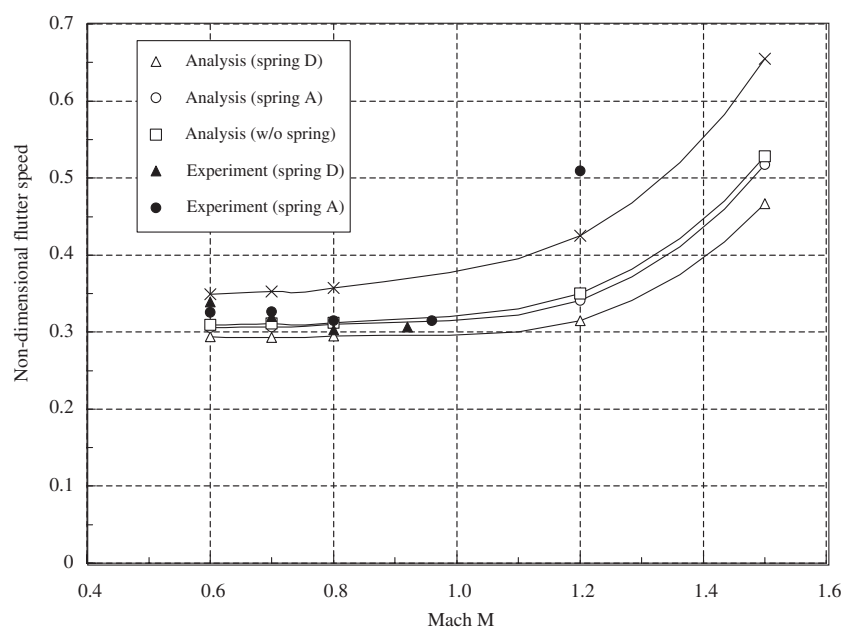

Fig. 9. Flutter boundary of anti-symmetric mode flutter.

\subsubsection{Correction in flutter calculation}

The flutter boundary obtained by the experiment becomes considerably higher at $M=1.2$ if it is compared with that of the analysis (Fig. 9). The similar result was obtained in the different studies using the same wing. ${ }^{12)}$ Flutter boundaries became higher in a low supersonic regime. ${ }^{13)} \mathrm{We}$ assume that the Mach number of a channel flow different from the free stream may cause the difference in the flutter boundaries. In the following, flutter analysis attempting to correct with the measured local Mach number for the same wing is described.

The local Mach number is calculated by the static pressures and the total pressures measured at the wing since the Mach number cannot be directly measured. Static pressures $P_{1}$ are measured by four pressure sensors at the right wing, two of which are located at the $31.1 \%$ and $90.7 \%$ chord on a span $45.5 \mathrm{~mm}$ inboard, and the other two are at 


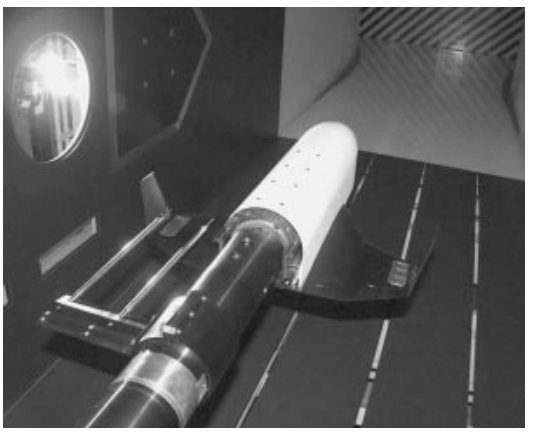

Fig. 10. Wind tunnel model, Pitot tube and pressure sensor.

the chord of $31.1 \%$ and $81.5 \%$ on a span $140.5 \mathrm{~mm}$ outboard. Total pressures near the pressure sensors are measured by a quartz manometer and by two Pitot tubes which can be manually traversed. In a supersonic regime, total pressures are measured as $P_{02}$ which are behind shockwaves caused by Pitot tubes. The Pitot tubes are set at the left wing to avoid interactions with pressure sensors on the right wing. Spanwise positions of the Pitot tubes are collocated to pressure sensor positions. The wind tunnel model, the Pitot tubes and the pressure sensors are illustrated in Fig. 10. Total and static pressures are measured in a steady flow condition with Mach numbers of 0.6, 0.8, 1.0, 1.1, 1.2, 1.3 and 1.4. Total pressures $P_{0}$ in a plenum chamber are $80 \mathrm{kPa}$ and $100 \mathrm{kPa}$.

A relationship between the local Mach number $M_{1}, P_{1}$ and $P_{02}$ is given by Eq. (2) which is based on the Rankine-Hugoniot relation. ${ }^{14)}$

$$
\frac{P_{1}}{P_{02}}=\frac{P_{1}}{P_{2}} \frac{P_{2}}{P_{02}}=\frac{\left(\frac{2 \gamma}{\gamma+1} M_{1}^{2}-\frac{\gamma-1}{\gamma+1}\right)^{\frac{1}{\gamma-1}}}{\left(\frac{\gamma+1}{2} M_{1}^{2}\right)^{\frac{1}{\gamma-1}}}
$$

The local Mach number $M_{1}$ is calculated by the Regula-Falsi method from measured $P_{1}$ and $P_{02}$ in a supersonic regime. On the other hand, the local Mach number for a subsonic regime is calculated by Eq. (3).

$$
\frac{P_{1}}{P_{0}}=\left(\frac{1}{1+(\gamma-1) M_{1}^{2} / 2}\right)^{\frac{\gamma}{\gamma-1}}
$$

The local Mach numbers $M_{1}$ are obtained at each location of the pressure sensors. However, a representative Mach number is required for the flutter analysis. The flutter couples with the 3 rd and 5th natural vibration modes where the deflection is larger in the outboard wing than inboard. Therefore, the local flow outboard has larger effects on the unsteady aerodynamics. A flutter analysis is conducted using the corrected Mach number $M^{\prime}$ that is the average of two pressures in the outboard wing as shown in Table 3. The analytical boundary obtained is illustrated in Fig. 11.

\subsubsection{Discussion}

In a roll free condition, the anti-symmetric flutter boundary is lower than that of the symmetric flutter. It was critical in both the experiment and the analysis. The analysis shows that this anti-symmetric flutter couples in the 3rd and 5th
Table 3. Corrected Mach number.

\begin{tabular}{cc}
\hline $\begin{array}{c}\text { Mach number } \\
\text { in free stream }\end{array}$ & $\begin{array}{c}\text { Corrected } \\
\text { Mach number }\end{array}$ \\
$M$ & $M^{\prime}$ \\
\hline 0.6 & 0.68 \\
0.8 & 0.93 \\
1.0 & 1.30 \\
1.1 & 1.35 \\
1.2 & 1.46 \\
1.3 & 1.61 \\
1.4 & 1.76 \\
\hline
\end{tabular}

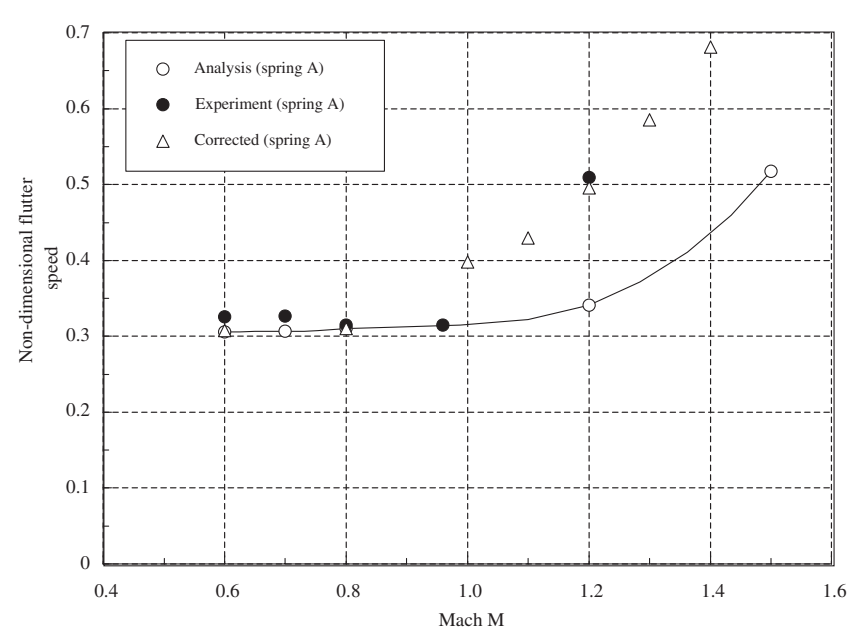

Fig. 11. Flutter boundary with correction.

natural vibration modes. As for the effects of the elastic rolling mode, the rolling stiffness lowers the flutter speed in the anti-symmetric flutter. This is due to the changes of the 1st and 3rd natural vibration modes because a rolling stiffness remarkably alters the characteristics of these natural vibration modes.

Next, we consider the difference between the analysis and the experiment at $M=1.2$. The experimental flutter speeds have become higher than the analytical one. They, however, agree favorably with the analysis that is corrected by the local Mach number. The difference is due to the local Mach number which is higher than that in the free stream. The difference becomes appreciable because the flutter speed increases as the Mach number is increasing in a supersonic regime.

\section{Effects of an Elastic Yawing Mode}

In this section, flutter characteristics affected by an elastic yawing mode will be presented. The tip-fin wing had been designed in the HOPE-X project. Large unsteady aerodynamic forces will act on the wing and will affect flutter characteristics because an elastic yawing mode considerably changes an angle attack for the tip-fin wing. The flutter coupling with an elastic yawing mode may occur even in a case of a planar wing. ${ }^{5,6)}$ 


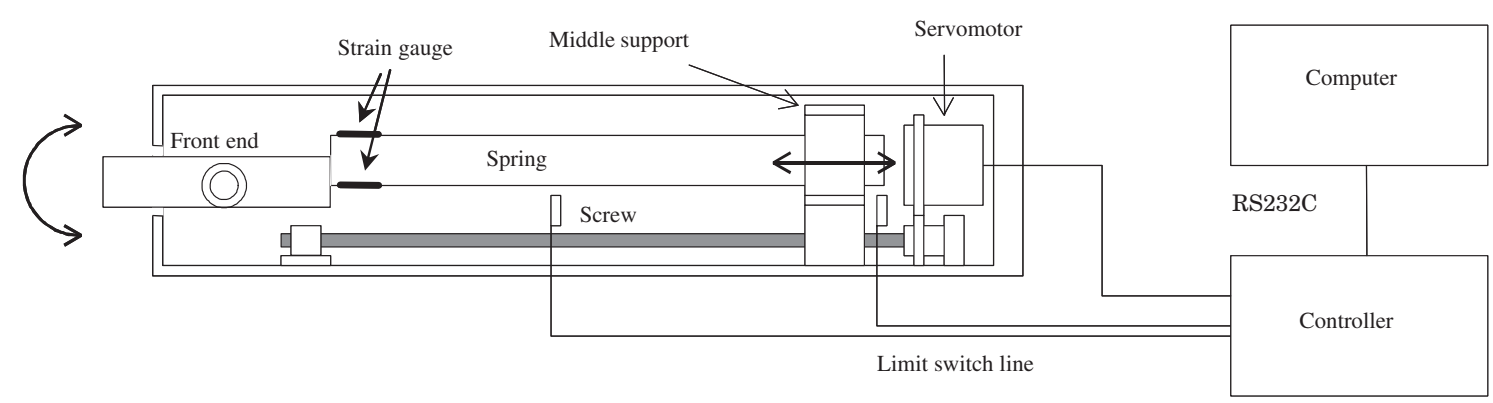

Fig. 12. Schematic of supporting system with elastic yawing mode.

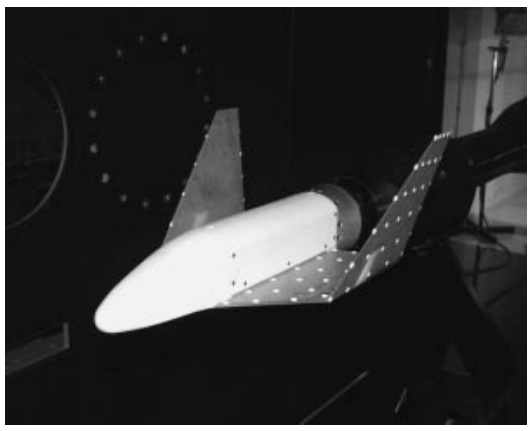

Fig. 13. Elastic yawing model.

A flutter experiment with the tip-fin model is conducted in the wind tunnel. The supporting system described above with an elastic yawing mode can simulate the launching configuration. The model also has an elastic rolling mode inside it. The flutter is also analytically studied.

\subsection{Supporting system and wind tunnel model}

\subsubsection{Supporting system}

The previous supporting system allowing elastic pitching motion $^{5,6)}$ is utilized for an elastic yawing mode by rotating 90 degrees. The configuration of the supporting system is shown in Fig. 12. The bending of the spring inside the support gives the yawing stiffness. A wind tunnel model is attached to one end of this spring, on which strain gauges are instrumented.

Further, the supporting system has a function to change the yawing stiffness. The spring inside the support is cantilevered at the front end and a middle support can be moved by a servomotor. Yawing stiffness changes from $K=$ 20,000 to $50,000 \mathrm{Nm} / \mathrm{rad}$ depending on the location of the middle support. $K$ is a rotational stiffness given by the differential bending of the springs. This function works as a flutter suppressor by changing the yawing stiffness when the flutter couples with an elastic yawing mode.

\subsubsection{Wind tunnel model}

Figure 13 shows a full setup (elastic yawing model). The wing consists of a main wing and two tip-fins. The tip-fin is intentionally larger than the real design in order to investigate the coupling of an elastic yawing mode. The model has strain gauges at the root of the main wing to measure the bending and torsion.

The fuselage is $455 \mathrm{~mm}$ in length and $86.8 \mathrm{~mm}$ in both height and width. The wing is made of aluminum alloy
7075-T651. The main wing is $5 \mathrm{~mm}$ in thickness, $100 \mathrm{~mm}$ in span length, $300 \mathrm{~mm}$ in chord length at the root and $220 \mathrm{~mm}$ at the tip. The tip-fin is $2 \mathrm{~mm}$ thick and has a cant angle of $14^{\circ}$, a span length of $204 \mathrm{~mm}$ and a chord length of $60 \mathrm{~mm}$ at the tip. There exists a $50 \%$ slit in the direction of the chord between the main wing and the tip-fin. The half wing weighs $0.52 \mathrm{~kg}$.

This model has an elastic rolling mode, which is provided by ball bearings and springs with a stiffness of $250 \mathrm{Nm} / \mathrm{rad}$. Strain gauges are attached on the springs.

\subsection{Modal characteristics}

\subsubsection{Modal tests}

A modal test has been conducted with the Dynamic Displacement Measurement System. Natural vibration modes are calculated from the measured dynamic displacements of 117 points (54 on each wing, 8 on the fuselage and one shaking point). The main wing was excited by an electromagnetic shaker with random signals of white noise. It was difficult to activate an elastic yawing mode because the direction of excitation was normal to the main wing. Therefore, an elastic yawing mode has been measured using the hammer impact method.

\subsubsection{Modal analysis}

Modal parameters are calculated by the FEM. The FEM model of the main wing and the tip-fin consist of shell elements. An elastic rolling mode is included by adding the torsional stiffness of a beam element and yawing is included by adding rotational flexibility to a beam for the sake of simplicity.

\subsubsection{Discussion}

Modal characteristics with the yawing stiffness of $K=$ $45,000 \mathrm{Nm} / \mathrm{rad}$ are shown in Table 4. Natural frequencies of the elastic yawing mode (the 4th mode) are shown in Table 5.

Compared with the test results, the analytical results have only a 5\% difference above the 5 th mode. Natural vibration modes and frequencies agree favorably in general. Analytical natural vibration modes and experimental natural frequencies are used for the flutter calculations.

\subsection{Flutter characteristics}

\subsubsection{Flutter experiments}

A flutter experiment has been conducted in the transonic wind tunnel. The model set in the wind tunnel is illustrated in Fig. 14. Flutter measurements were made while the total pressure $P_{0}$ was increased step by step for the Mach num- 
Table 4. Modal characteristics of elastic yawing model.

\begin{tabular}{cccl}
\hline \multirow{2}{*}{ Number } & \multicolumn{2}{c}{ Frequency $[\mathrm{Hz}]$} & \\
\cline { 2 - 3 } & Experiment & Analysis & \\
\hline 1 & 18 & 19 & Elastic rolling \\
2 & 29 & 28 & 1st symmetric tip-fin bending \\
3 & 33 & 32 & 1st anti-symmetric tip-fin bending \\
4 & 56 & 56 & Elastic yawing \\
5 & 108 & 102 & 1st symmetric tip-fin torsion \\
6 & 108 & 102 & 1st anti-symmetric tip-fin torsion \\
7 & 137 & 141 & 2nd symmetric tip-fin bending \\
8 & 166 & 164 & 2nd anti-symmetric tip-fin bending \\
9 & - & 170 & Symmetric tip-fin mode \\
\hline
\end{tabular}

Table 5. Natural frequency of elastic yawing mode.

\begin{tabular}{ccc}
\hline Yawing elasticity & \multicolumn{2}{c}{ Frequency $[\mathrm{Hz}]$} \\
\cline { 2 - 3 }$[\mathrm{Nm} / \mathrm{rad}]$ & Experiment & Analysis \\
\hline 20000 & 45 & 45 \\
25000 & 49 & 49 \\
30000 & 50 & 50 \\
35000 & 52 & 52 \\
40000 & 54 & 54 \\
45000 & 56 & 56 \\
50000 & 58 & 58 \\
\hline
\end{tabular}

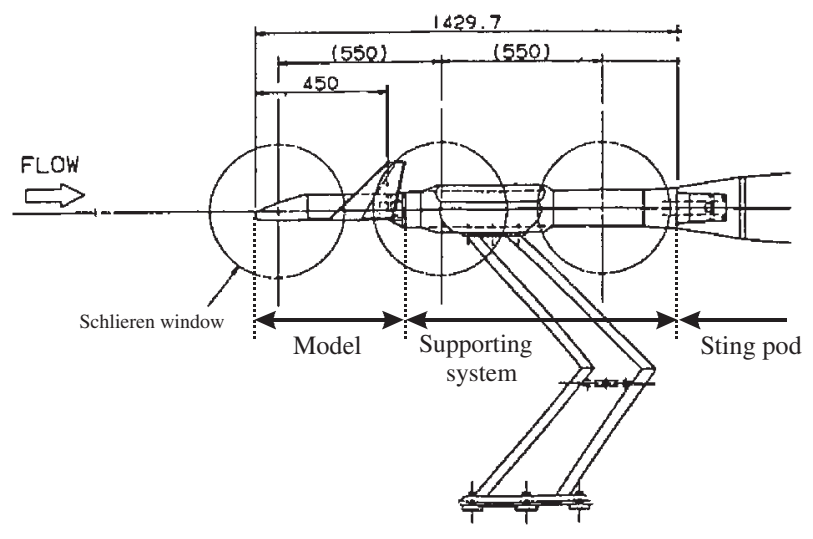

Fig. 14. Wind tunnel test.

bers of $0.6,0.7$, and 0.8 . The function of the supporting system was employed to suppress flutter. As a result, flutter points are obtained at each Mach number selected. Figure 15 shows the time histories of strain at $M=0.6$ with the yawing stiffness of $K=45,000 \mathrm{Nm} / \mathrm{rad}$. The left figures show the time histories from onset of flutter to its suppression. The right ones show the time histories during the flutter. This flutter is anti-symmetric with respect to the plane of symmetry and the strain due to the yawing is large. We call this flutter a 'yawing mode flutter', which has never appeared in the past.

It was rather difficult to determine flutter boundaries. One of the reasons is that the flutter boundaries varied by the repeated operation for the yawing stiffness change. The other reason is that the oscillation could not be easily judged if it

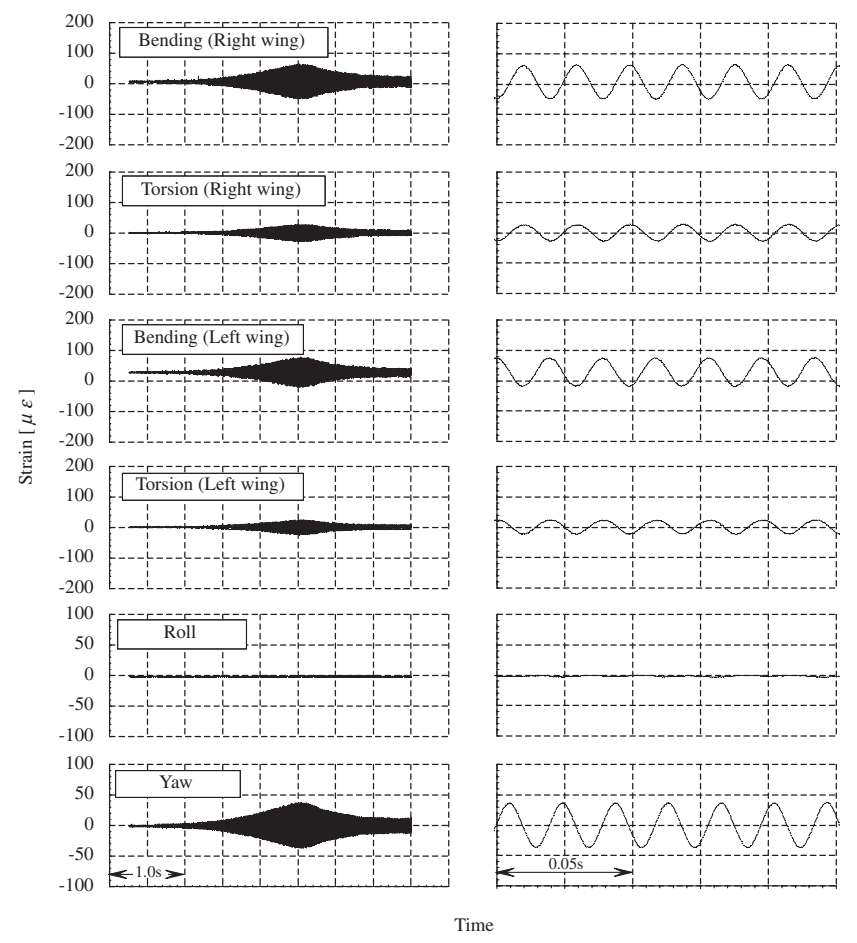

Fig. 15. Time history of yawing mode flutter.

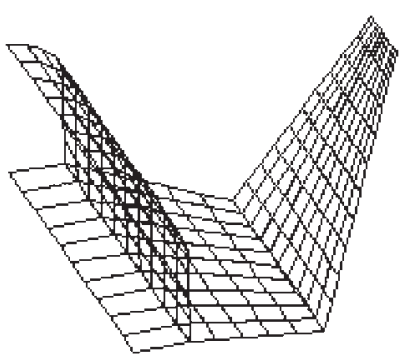

Fig. 16. Aerodynamic model.

diverges or not. Flutter boundaries seem to be sensitive to the structural damping. The structural damping varies due to the mechanism of the supporting system. Representative flutter boundaries were determined with multiple runs at the same condition.

\subsubsection{Flutter analysis}

Theoretical analysis has also been conducted for the flutter. The method employed is the same as shown in Sec. 2. The aerodynamic panels consist of 240 elements (50 for the main wing, 100 for the tip-fin and 90 for the fuselage) in the right half of the model as shown in Fig. 16. The 1st, 3 rd, 4th and 6th modes are used to compute the anti-symmetric unsteady aerodynamic forces while the 2nd, 5th, 7 th and 9th modes are for the symmetric forces. The structural damping applied is 0.0, 0.005, 0.01 and 0.02 .

As a result, three kinds of flutter have been obtained; (1) flutter coupling with the $3 \mathrm{rd}$ and the 4 th mode (yawing mode flutter), (2) symmetric flutter coupling with the 2 nd and the 5 th mode, (3) anti-symmetric flutter coupling with the $3 \mathrm{rd}$ and the 6th mode. As an example, the analytical result of the yawing mode flutter with a yawing stiffness of $K=$ 

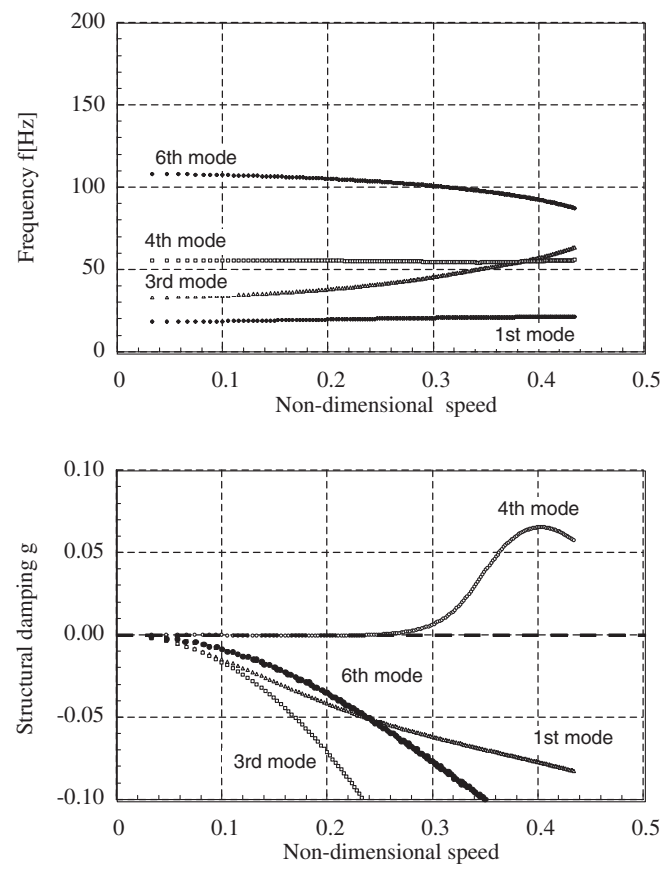

Fig. 17. Yawing mode flutter analysis.

$45,000 \mathrm{Nm} / \mathrm{rad}$ at $M=0.6$ is shown in Fig. 17. The non-dimensional flutter speed depending on the structural damping at $M=0.6$ is shown in Fig. 18. Boundaries of symmetric and anti-symmetric flutter coupling with a bending and a torsional mode do not depend much on the structural damping. However, the yawing mode flutter boundary varies greatly and is sensitive to the structural damping.

\subsubsection{Discussion}

Yawing mode flutter points in the experiment and in the analysis with the yawing stiffness of $K=40,000$ and $45,000 \mathrm{Nm} / \mathrm{rad}$ are shown in Fig. 19. The structural damping is 0.01 in the analysis. Analytical flutter points with the varying structural damping between 0.00 and 0.02 are also shown with the vertical lines where the maximum speed is for the damping of 0.02 and the minimum for 0.00 in the same line.

A quantitative comparison seems to be difficult since the structural damping is thought to be changing due to the

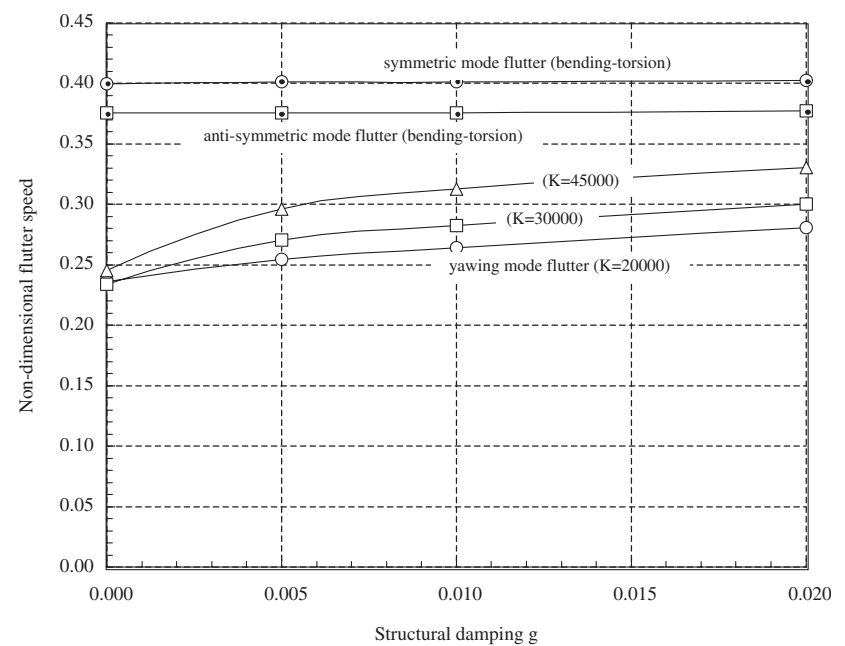

Fig. 18. Flutter boundary as function of structural damping.

mechanism of the supporting system. The flutter points with the damping of 0.01 reasonably agree with each other. We can also recognize from the experiments and the analysis that the yawing mode flutter actually becomes critical and that its flutter boundary varies with the yawing stiffness. This type of flutter is sensitive to the structural damping. They have fairly good agreements.

\section{Concluding Remarks}

Flutter characteristics of a winged reentry space vehicle in launching configuration have been studied in this paper. Flutter experiments and analyses have been conducted for a vehicle model having an elastic rolling mode or elastic yawing one, assuming an attachment between the space vehicle and the rocket.

In the case of an elastic rolling model, the anti-symmetric flutter coupling with the bending and torsion becomes critical. An elastic rolling mode lowered the flutter speed. On the other hand, the yawing model gives the critical flutter that involves an elastic yawing mode. The yawing mode flutter is a coupling between the elastic yawing mode and the anti-symmetric bending mode. This type of flutter is
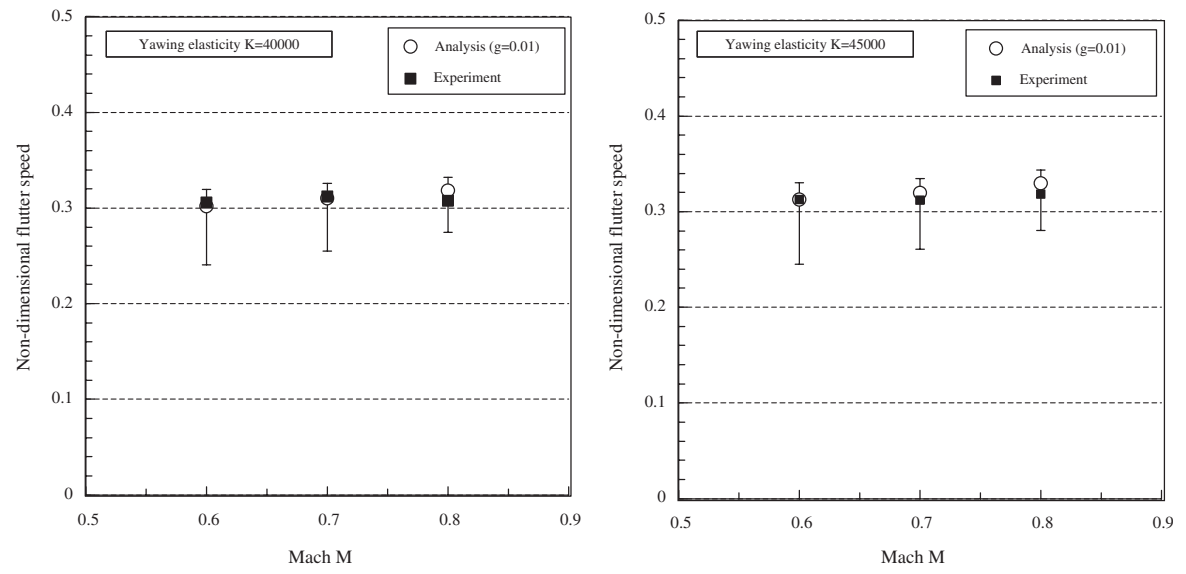

Fig. 19. Flutter boundary of yawing mode flutter. 
rather sensitive to the structural damping.

Flutter characteristics involving the elastic rolling or the elastic yawing mode have been clarified. These types of flutter can be critical. In the configuration of the vehicle mounted atop a rocket like HOPE-X, the flutter, including the elastic modes of the attachment, should be considered in the design.

\section{References}

1) Perry III, B. and Cole, S. R.: Summary of an Active Flexible Wing Program, J. Aircraft, 32 (1995), pp. 10-15.

2) Soistmann, D. L. and Spain, C. V.: An Experimental and Analytical Study of a Lifting-Body Wind-Tunnel Model Exhibiting Body-Freedom Flutter, AIAA Paper 93-1316, 1993.

3) Chen, G. S. and Dugundji, J.: Experimental Aeroelastic Behavior of Forward-Swept Graphite/Epoxy Wings with Rigid-Body Freedom, J. Aircraft, 24 (1987), pp. 454-462.

4) Niblett, L. T.: The Fundamentals of Body-Freedom Flutter, Aeronaut. J., 90 (1986), pp. 373-377.

5) Kanda, A. and Ueda, T.: Wind Tunnel Tests and Analysis on Flutter of Spacecraft Including Pitching Effects in Its Launching Configuration, International Forum on Aeroelasticity and Structural Dynamics, Wil- liamsburg, Virginia, 1999, pp. 115-120.

6) Kanda, A. and Ueda, T.: Flutter Characteristics of Winged Vehicle in Free-Flight/Launching Configuration and Development of Model Supporting System, J. Jpn. Soc. Aeronaut. Space Sci., 49 (2001), pp. 346-353 (in Japanese).

7) Ueda, T.: Unsteady Aerodynamic Calculations for General Configurations by the Doublet-Point Method, Technical Report of National Aerospace Laboratory, TR-1101T, 1991.

8) Kanda, A. and Ueda, T.: Sting Supporting System with Controllable Roll Mode, NAL TR-1335, 1997 (in Japanese).

9) Sotozaki, T. and Ueda, T.: Preliminary Vibration Tests Using the Dynamic Displacement Measurement System, NAL TM-683, 1995 (in Japanese).

10) Kanda, A. and Sotozaki, T.: Replacement of Dynamic Displacement Measurement System, NAL TM-748, 2000 (in Japanese).

11) Bisplinghoff, R. L. and Ashley, H.: Principle of Aeroelasticity, John Wiley \& Sons, New York, 1962, pp. 235-258.

12) Kanda, A. and Ueda, T.: Anti-Symmetric Mode Flutter of Winged Reentry Space Vehicle, NAL TR-1342, 1997 (in Japanese).

13) Isogai, K., Ejiri, H., Kikuchi, T., Nakamichi, J., Noguchi, Y. and Morita, K.: Experimental Study on Transonic Flutter Characteristics of Sweptback Wing with Core Composite Plates Having Different Fiber Orientations, NAL TR-827, 1984 (in Japanese).

14) Aihara, Y.: Nagare no Rikigaku, Baifukan, Tokyo, 1990, pp. 33-42 (in Japanese). 